\title{
Energy migration in molecular assemblies: The characterisation and differentiation of two-photon mechanisms
}

\author{
David S. Bradshaw and David L. Andrews* \\ Nanostructures and Photomolecular Systems, School of Chemical Sciences, \\ University of East Anglia, Norwich NR4 7TJ, U.K.
}

\begin{abstract}
Energy migration between chromophores plays a prominent role in a range of energy harvesting assemblies. Recent advances in the design and production of light-harvesting polymers have led to the synthesis of novel two-photon absorbing dendrimers. To construct increasingly efficient multifunctional macromolecules of this type, understanding the inherent optical processes and disentangling them has become imperative. This paper explores the fundamental processes by means of which energy transfers from a donor chromophore to an acceptor through two-photon absorption from an input laser beam. It is determined that three distinct classes of mechanism can operate: (i) two-photon absorption by individual chromophores is followed by transfer of the energy to an acceptor group; (ii) a singly excited chromophore is excited to a virtual state by the additional absorption of a photon from the pump radiation field, coupled with resonance energy transfer to the acceptor, or; (iii) two-photon excitation of the acceptor results from acquisition of one quantum of energy from a singly excited neighbour group and another from the throughput radiation. These mechanisms may compete and, in certain cases, lead to manifestations of quantum interference. Generally, the most favoured mechanism is determined by a balance of factors and constraints. Principal amongst the latter are the choice of wavelength (connected with the possibility of exploiting certain electronic resonances, whilst judiciously avoiding others) and the precise chromophore architecture (taking account of geometric factors concerned with the relative orientation of transition moments). As the relative importance of each mechanism determines the key nanophotonic characteristics of the assembly, the principles and results reported here afford the means for expediting highly efficient two-photon energy migration.
\end{abstract}

Keywords: Energy migration, nanophotonics, two-photon absorption, nonlinear optics, light harvesting, dendrimers

\section{INTRODUCTION}

In the evolving technology of photoactive materials, much of the effort in the design and synthesis of multifunctional polymers and other molecular assemblies is being directed towards increasing photon capture and energy harvesting efficiency. Typically, energy acquired as a result of photon absorption migrates to a core through a sequence of energy transfer events, and efficiency gains are largely a result of optimising and exploiting features of the transfer mechanism. In any single instance of the latter, energy transfer is generally mediated by a coupling between transition electric dipole moments of two chromophore species playing the transient role of donor and acceptor - a mechanism usually known as Förster resonance energy transfer. Over any distance beyond wavefunction overlap, this is the key factor in a wide range of phenomena including natural light-harvesting ${ }^{1,2}$ - especially energy hopping between chlorophyll molecules in the photosynthetic unit. It is also a feature of several other technical applications, including methods for nanoscale structure determination ${ }^{3,4}$ (such as the use of the popularly termed 'spectroscopic ruler' to deliver information on intramolecular distances in proteins) and nonlinear optics - particularly frequency conversion in materials such as lanthanide-doped crystals. ${ }^{5-7}$ Recent advances in the production and characterisation of synthetic light-harvesting polymers have led to the identification of two-photon absorbing dendrimers, ${ }^{8-16}$ the latter term signifying multiplybranched macromolecules of essentially fractal geometry. With a host of potential applications ranging from optical switching to photodynamic cancer therapy, such optically nonlinear systems are attracting keen attention and there is an urgent need to clarify the different mechanisms that come into play when two photons are involved. This is the principal aim of the present paper. 


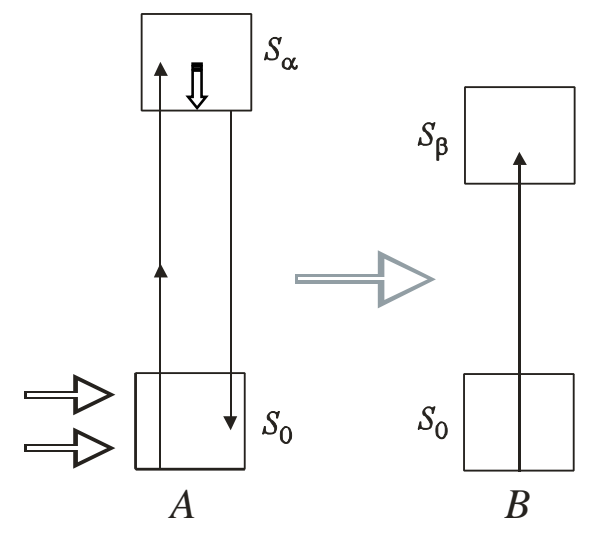

Figure 1. Energy scheme for TPRET: $S_{0}$ represents the donor ground electronic state and its vibrational manifold; $S_{\alpha}$ and $S_{\beta}$ denote the higher electronic states (and their associated manifolds) of donor $A$ and acceptor $B$, respectively. Black and grey vertical arrows represent input beam and transfer photons, respectively; horizontal arrows denote channels of excitation transfer.

We examine a fundamental unit, within a multichromophore system, comprising a donor $A$ and an acceptor $B$, irradiated by an input laser beam from which two photons are absorbed. The acceptor is generally assumed to be optically transparent at the input (and the second harmonic) frequency. In comparison to one-photon absorbing systems, where only one type of transfer mechanism can be envisaged, two-photon absorption (TPA) introduces the possibility of three distinct classes of competing processes - these are to be closely investigated in the following. [Note, mechanisms (foreseen by ref. 17) where the system emits a photon back into the throughput beam are not considered in the present context of a multichromophore assembly, since the subsequent migration of a single photon energy presents no intrinsically distinctive features. Nonetheless such processes are of interest in the context of optical switching, since they offer the means to effect a novel optical form of transistor action]. ${ }^{18-20}$

The first process to come under scrutiny is a two-photon resonance energy transfer (TPRET) mechanism. Here, as illustrated by the energy scheme of figure 1, donor $A$ absorbs two photons from the input beam and the transfer of energy to the acceptor follows. ${ }^{21,22}$ This can be expressed by the following chemical representation;

$$
A+B+2 \hbar \omega \rightarrow A^{* *}+B \rightarrow A+B^{*}
$$

where the two-star superscript denotes a two-photon excited state of the donor. A second process, to be termed the antiStokes electronic Raman-induced transfer (ASERIT), proceeds through single photon absorption by the donor to populate an excited state. By the further absorption of a laser input photon the donor is then promoted to a virtual state whose decay couples with the acceptor excitation through resonance energy transfer. This process is given by the representation;

$$
A+B+2 \hbar \omega \rightarrow A^{*}+B+\hbar \omega \rightarrow A+B^{*}
$$

and is schematically shown in figure 2. The third process, to be labelled transfer-induced double-quantum excitation (TIDQE), entails two-quantum excitation of the acceptor by acquisition of a quantum of energy from a single-photon excited donor and another photon from the throughput radiation. This mechanism is identified with the same chemical representation as ASERIT, but its depiction in figure 3 shows its clear mechanistic difference. The chemical equation is the same because both processes lead through the same progression of physically realisable states, but the transition from the intermediate state to the final state occurs through different virtual states (energy non-conserving states which are not sustained into or beyond the femtosecond timescale). The ASERIT and TIDQE mechanisms, which were first recognised by Stockmann, ${ }^{23}$ require pump radiation to be present in the molecular system after the initial excitation, in order to fulfil the conditions for the intermediate states. Such processes do not arise in conventionally configured experiments based on pump-probe laser techniques. 


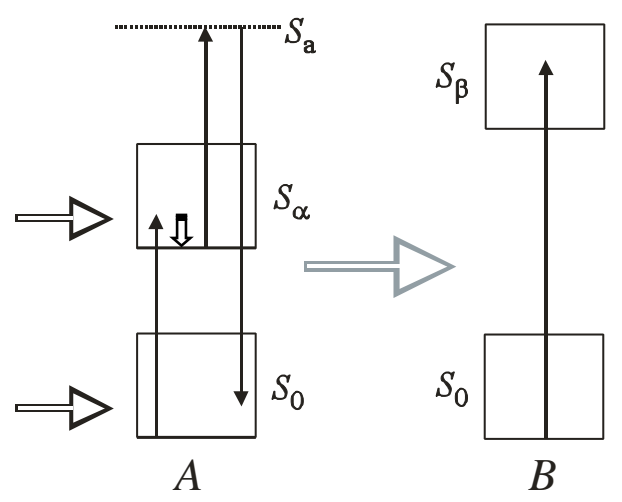

Figure 2. Energy scheme for ASERIT: $S_{\text {a }}$ denotes a virtual state within donor $A$.

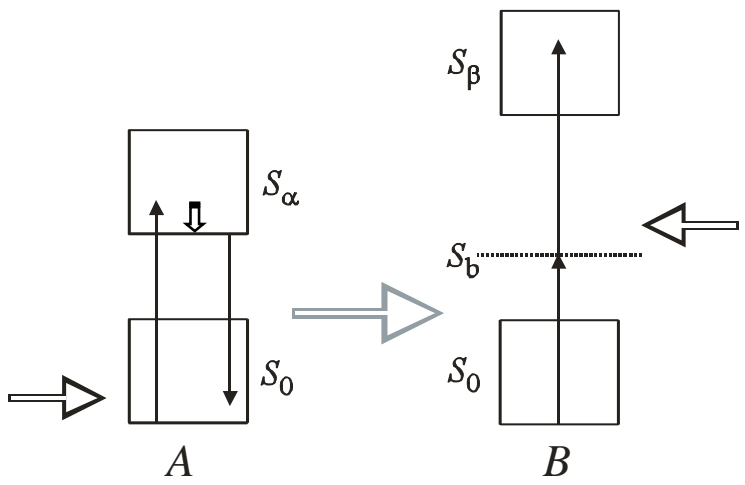

Figure 3. Energy scheme for TIDQE: $S_{\mathrm{b}}$ represents a virtual state within acceptor $B$.

\section{THEORETICAL REPRESENTATIONS OF THE MECHANISMS}

The three described processes are duly represented by a theory based on the principles of quantum electrodynamics (QED). To aid the calculations, Feynman diagrams are constructed for each of these mechanisms (figures 4-6). For clarity, let us define the concerted part of the process as the portion that begins and ends at real states where dissipation through intramolecular vibrational redistribution (IVR) may arise, traversing only virtual states between them. On application of Fermi's golden rule, ${ }^{24}$ a rate for each concerted part of the three processes is found. For the TPRET mechanism (figure 4) the rate equation for the first part, namely the initial two-photon absorption by the donor, is given by ${ }^{25}$

$$
\Gamma_{\mathrm{TPA}}=\frac{\pi}{8 \hbar}\left(\frac{I}{\varepsilon_{0} c}\right)^{2}\left|e_{i} \alpha_{i j}^{\alpha 0(A)}\left(\omega_{0}, \omega_{0}\right) e_{j}\right|^{2} \rho_{f}^{A^{* *}}
$$

where $I$ is the irradiance, e denotes the polarisation unit vector, $\omega_{0}$ symbolises the optical input circular frequency and $\rho_{f}^{A^{* * *}}$ is the excited state density of states. Further, the general expression for the polarisability tensor, $\alpha_{i j}$, is given by; 


$$
\alpha_{i j}^{f(\xi)}\left(\mp \omega_{1}, \mp \omega_{2}\right)=\sum_{\zeta}\left\{\frac{\mu_{i}^{f \zeta(\xi)} \mu_{j}^{\zeta i(\xi)}}{E_{i \zeta} \pm \hbar \omega_{1}}+\frac{\mu_{j}^{f \zeta(\xi)} \mu_{i}^{\zeta i(\xi)}}{E_{i \zeta} \pm \hbar \omega_{2}}\right\}
$$

where $i, \zeta$ and $f$ are the initial, virtual and final states respectively through which molecule $\xi$ progresses; also the transition dipole moment is given by $\boldsymbol{\mu}^{f(\xi)} \equiv\left\langle\xi^{f}\left|\boldsymbol{\mu}^{(\xi)}\right| \xi^{\zeta}\right\rangle$ and molecular transition energy by $E_{i \zeta}=E_{i}-E_{\zeta}$. The second concerted part is one-photon RET, which is represented by the rate equation; ${ }^{26}$

$$
\Gamma_{\mathrm{RET}}=\frac{2 \pi}{\hbar}\left|\mu_{k}^{0 \alpha(A)} V_{k l}\left(\omega^{\prime}, \mathbf{R}\right) \mu_{l}^{\beta 0(B)}\right|^{2} \rho_{f}^{B} .
$$

Here, $\omega^{\prime}$ is the donor emission frequency for which $\omega^{\prime} \leq 2 \omega_{0}$ to account for the dissipative effect of IVR, and $V_{k l}$ defines the electric dipole-electric dipole coupling tensor that is given by;

$$
V_{k l}\left(\omega^{\prime}, \mathbf{R}\right)=\frac{\exp \left(\frac{\mathrm{i} \omega^{\prime} R}{c}\right)}{4 \pi \varepsilon_{0} R^{3}}\left\{\left(\delta_{k l}-3 \hat{R}_{k} \hat{R}_{l}\right)-\left(\frac{\mathrm{i} \omega^{\prime} R}{c}\right)\left(\delta_{k l}-3 \hat{R}_{k} \hat{R}_{l}\right)-\left(\frac{\omega^{\prime} R}{c}\right)^{2}\left(\delta_{k l}-\hat{R}_{k} \hat{R}_{l}\right)\right\},
$$

with the donor-acceptor displacement vector denoted as $\mathbf{R}=\mathbf{R}_{B}-\mathbf{R}_{A}$. The complete TPRET rate equation is determined by a product of the probability of the first, two-photon absorption, part (which is the rate multiplied by the laser pulse duration $\tau_{L}$ ) and the rate of the second RET part, hence from (1) and (3) the following expression arises;

$$
\Gamma_{\text {TPRET }}=\left(\frac{\pi I}{2 \varepsilon_{0} \hbar c}\right)^{2} \tau_{L}\left|e_{i} \alpha_{i j}^{\alpha 0(A)}\left(\omega_{0}, \omega_{0}\right) e_{j}\right|^{2}\left|\mu_{k}^{0 \alpha(A)} V_{k l}\left(\omega^{\prime}, \mathbf{R}\right) \mu_{l}^{\beta 0(B)}\right|^{2} \rho_{f}^{A^{* * *}} \rho_{f}^{B} .
$$

Turning to the ASERIT mechanism (figure 5), a real (intrinsically lifetime-limited) state is populated after both the initial one-photon absorption at the donor and also following an intricate RET step (involving the absorption of a further input photon at $A$ ). Therefore, by multiplying each of the corresponding rate equations together with the lifetime of the real electronic state of molecule $A$ (denoted $\tau_{A}$ ) - the multiplication factor being due to the fact that the initial excitation depends on the pulse length of the input beam, $\tau_{L}$, while the following absorption depends on the fraction $\tau_{A} / \tau_{L}-$ the complete ASERIT rate equation is given by;

$$
\Gamma_{\mathrm{ASERIT}}=\left(\frac{\pi I}{\varepsilon_{0} \hbar c}\right)^{2} \tau_{A}\left|e_{i} \mu_{i}^{\alpha 0(A)}\right|^{2}\left|e_{j} \alpha_{j k}^{0 \alpha(A)}\left(-\omega, \omega_{0}\right) V_{k l}(\omega, \mathbf{R}) \mu_{l}^{\beta 0(B)}\right|^{2} \rho_{f}^{A^{*}} \rho_{f}^{B},
$$

where $\omega$ corresponds to the donor emission frequency, with $\omega \leq \omega_{0}$ due to excited state IVR. Similarly, the rate equation of the TIDQE mechanism (figure 6) is given by;

$$
\Gamma_{\mathrm{TIDQE}}=\left(\frac{\pi I}{\varepsilon_{0} \hbar c}\right)^{2} \tau_{A}\left|e_{i} \mu_{i}^{\alpha 0(A)}\right|^{2}\left|e_{j} \alpha_{j l}^{\beta 0(B)}\left(\omega, \omega_{0}\right) V_{k l}(\omega, \mathbf{R}) \mu_{k}^{0 \alpha(A)}\right|^{2} \rho_{f}^{A^{*}} \rho_{f}^{B} .
$$

On examining the equations (5), (6) and (7), it is noteworthy that each of the mechanisms exhibits the standard quadratic dependence on intensity that is the hallmark of two-photon absorption. In the next section the above expressions will serve as a basis for judging the relative efficiency of each process. 


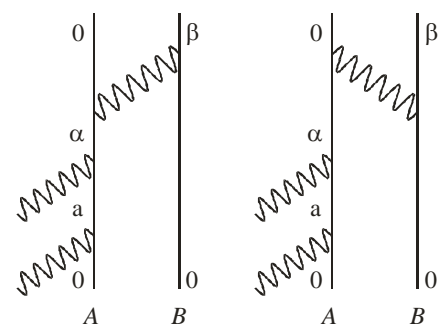

Figure 4. The two Feynmann diagrams for TPRET: 0 denotes the ground state level, $\alpha$ and $\beta$ are excited levels for molecules $A$ and $B$, respectively; the donor virtual state is denoted by a - for details see ref. 21 .
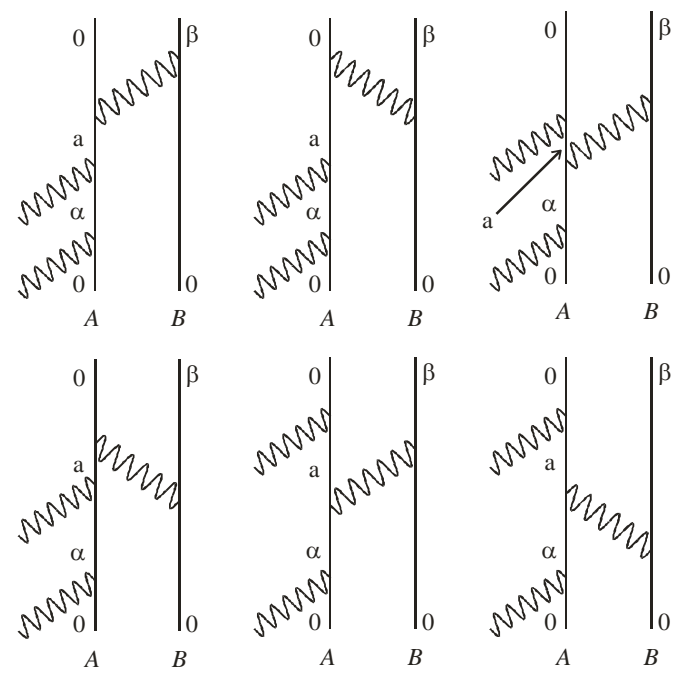

Figure 5. The six Feynmann diagrams of ASERIT: key as figure 4.

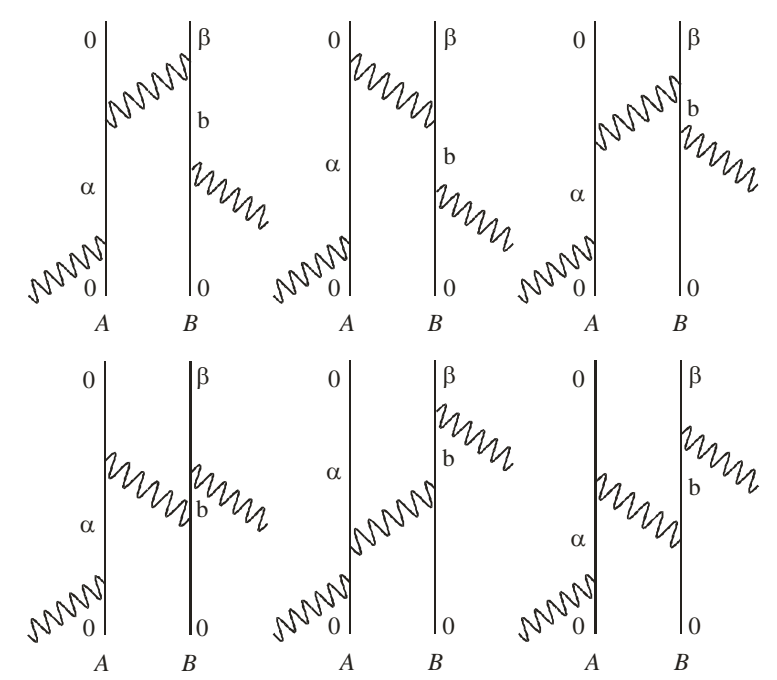

Figure 6. The six Feynmann diagrams of TIDQE: key as figure 4, and b denotes a virtual state of the acceptor. 


\section{RELATIVE EFFICIENCY OF EACH MECHANISM}

A starting point for an exploration of structural and electronic factors, and their bearing on the relative efficiencies of each mechanism, is the determination of a ratio involving equations (5), (6) and (7), i.e.;

$$
\gamma=\frac{\Gamma_{\mathrm{TPRET}}}{\Gamma_{\mathrm{TOT}}} \equiv \frac{\Gamma_{\mathrm{TPRET}}}{\Gamma_{\mathrm{TPRET}}+\Gamma_{\mathrm{ASERIT}}+\Gamma_{\mathrm{TIDQE}}} .
$$

It should be understood that such a notional ratio has a primarily representational meaning, and in many cases one or other of the possible excitation channels may be forbidden by selection rules, as we have discussed at length elsewhere (see ref. 22). The explicit form of this expression is highly intricate, so to pursue a readily comprehensible physical interpretation it is supposed that all transition dipole moments and $\alpha$ tensors (assume isotropic) are equivalent. Hence, in the short-range region, the simple result $\gamma \approx 1 / 9$ emerges. Specifically, under these conditions, the TPRET mechanism will be of smaller significance with respect to the other two processes by a ratio of 1:4:4. However, ASERIT and TIDQE may exhibit constructive or destruction quantum interference, deriving from the fact that they pass through the same real states (as observed earlier). In contrast, despite having the same initial and final states, TPRET may compete, but will not interfere with these mechanisms due to energy dissipation through differing channels of IVR in the donor molecule. Accommodating quantum interference, the ratio of equation (8) thus takes values between 1/17 (constructive interference) and 1 (destructive). ASERIT and TIDQE pathways from the initial to the final state of the system, along with the quantum interference of these pathways are schematically illustrated by figure 7 .

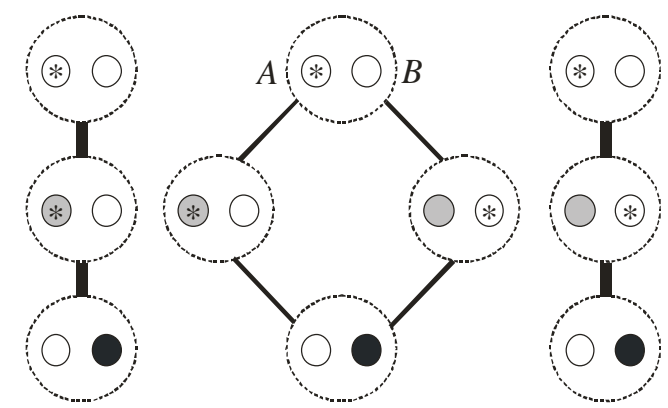

Figure 7. ASERIT and TIDQE pathways are illustrated left and right, respectively; centre depicts the quantum interference of these pathways. White, black and grey circles denote ground, excited and virtual states species respectively; asterisks represent the molecular centre at which the input photon is absorbed, and thick connecting lines signify single channel contributions; thin lines denote interfering channels. Here, the initial and final states are shown in the upper and lower boxes, respectively.

Further factors that determine the favoured mechanism can be resolved by detailed inspection of a specific energy level scenario (exemplified by figure 8). In the case illustrated here, a real excited state is not accessible after two-photon absorption by the donor and, hence, TPRET can be excluded from consideration. Nevertheless, a real state is accessed by one-photon excitation at $A$ and thus, following vibrational relaxation in that electronic excited state, the overall process could in principle continue to completion by both ASERIT and TIDQE (for simplicity, quantum interference is assumed negligible). Under the illustrated conditions, i.e. with energy levels spaced as shown, it does not appear that TIDQE can proceed any further, due to the fact that the energy sum of the two absorbed photons at the acceptor is less than $E_{\beta 0}$. Nonetheless, by exploiting thermal excitation of higher vibrational levels within the ground state manifold of the acceptor, TIDQE can become energetically permitted. The efficiency of this mechanism is further enhanced if the energy of either absorbing photon matches a real molecular state, in which case the process corresponds to one-photon absorption at both donor $A$ and acceptor $B$, and one-photon resonance energy transfer. The ASERIT mechanism is increasingly prominent for smaller values of $\Delta E \equiv E_{\alpha \zeta}-\hbar \omega_{0}$, since this results in the $\alpha$ tensor of equation (6) escalating in magnitude (as follows from equation (2)). For $\Delta E \approx 0$, however, it is necessary to include a resonance damping factor $\hbar \Gamma$ within the denominators of (2). One can conclude that ASERIT is at its most important when $\Delta E \approx$ $\hbar \Gamma$; for example, with $\Gamma=100$ fs the value of $\Delta E$ will be $6.6 \times 10^{-48} \mathrm{~J}$. 


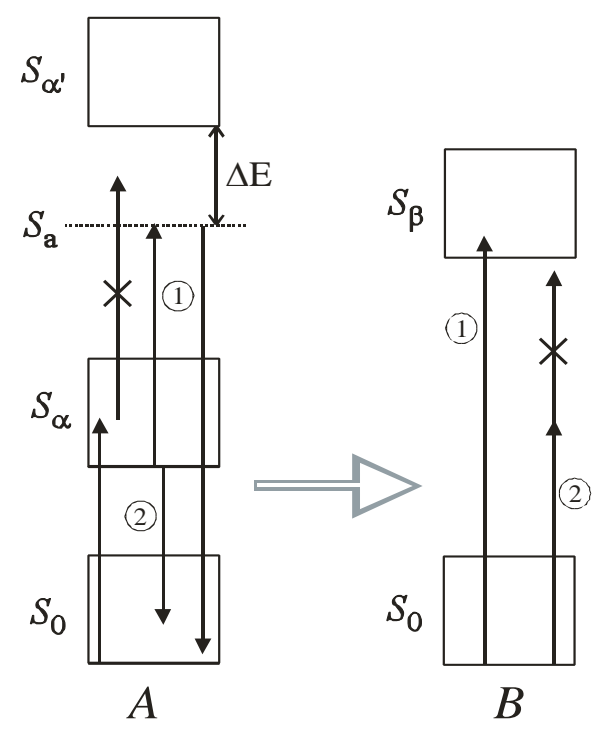

Figure 8. Illustrative scheme of molecular energy levels for which only the ASERIT mechanism is the allowed process. Here, (1) and (2) respectively denote ASERIT and TIDQE routes through the energy levels (following one-photon excitation).

Before concluding, it is worth noting that there are other mechanisms which might come into play through bimolecular interactions, associated with synergetic processes in which cooperative and distributive mechanisms lead to a final state where both donor and acceptor chromophores are excited. ${ }^{27}$ Although ostensibly similar in nature to ASERIT and TIDQE, respectively, they significantly differ in the constraints they impose on laser wavelength. Moreover, all the intermediate molecular states that participate in those mechanisms are virtual in nature; accordingly, the overall mechanisms are likely to occur with significantly lower levels of efficiency. The effect of other chromophores might also need to be taken into account. For example, by inclusion of another donor in the molecular system a further mechanism may compete, which is termed three-body energy pooling. ${ }^{28-30}$ Here, following initial one-photon excitations of two donors, collective pooling of these energies to an acceptor may occur. Once again, a distinction can be drawn on the basis of the very different selection rules which apply in such cases (see ref. 22). In general, the combined constraints of energy level placement and selection rules will be such that one or other of the possible mechanisms can usually be excluded from consideration.

\section{CONCLUSION}

We have exhibited the operation of the various conditions and constraints that can operate when molecular assemblies acquire the energy of two photons from throughput optical radiation. We have illustrated those principles through the example of one particular case in which ASERIT represents the only viable mechanism for the delivery of two photon energies to the acceptor. We have also drawn attention to the interesting property that supplying heat to such a system allows it to access the TIDQE mechanism, and that the two mechanisms may in principle interfere. The result suggests that distinctive changes in the efficiency of populating the resulting highly excited electronic state of the acceptor (manifest by changes in the corresponding short-wavelength fluorescence signal) may result from such thermal activation. A variety of other cases exhibiting quite different behavior are possible, and they are the subject of ongoing investigation.

\section{ACKNOWLEDGEMENTS}

This research from the QED group at the University of East Anglia is funded by the UK Engineering and Physical Sciences Research Council. 


\section{REFERENCES}

1. T. Renger, V. May and O. Kühn, "Ultrafast excitation energy transfer dynamics in photosynthetic pigment-protein complexes", Phys. Rep. 343, pp. 137-254, 2001.

2. R. J. Cogdell, N. W. Isaacs, A. A. Freer, T. D. Howard, A. T. Gardiner, S. M. Prince and M. Z. Papiz, “The structural basis of light-harvesting in purple bacteria", FEBS Lett. 555, pp. 35-39, 2003.

3. C. G. dos Remedios and P. D. J. Moens, pp. 1-64 in Resonance Energy Transfer, eds D. L. Andrews and A. A. Demidov, Wiley, Chichester, 1999.

4. B. Schuler, E. A. Lipman, P. J. Steinbach, M. Kumke and W. A. Eaton, "Polyproline and the 'spectroscopic ruler' revisited with single-molecule fluorescence”, Proc. Natl. Acad. Sci. USA 102, pp. 2754-2759, 2005.

5. V. V. Ovsyankin, pp. 343-481 in Spectroscopy of Solids Containing Rare Earth Ions, eds A. A. Kaplyanskii and R. M. Macfarlane, Elsevier, Amsterdam, 1987.

6. K. D. Oskam, R. T. Wegh, H. Donker, E. V. D. van Loef and A. Meijerink, "Downconversion: a new route to visible quantum cutting", J. Alloys Comps. 300-301, pp. 421-425, 2000.

7. C. Z. Hadad and S. O. Vasquez, "Statistical approach to the transient up-converted population in monodoped amorphous solids", Phys. Chem. Chem. Phys. 5, pp. 3027-3033, 2003.

8. A. Adronov, J. M. J. Fréchet, G. S. He, K. S. Kim, S. J. Chung, J. Swiatkiewicz and P. N. Prasad, "Novel two-photon absorbing dendritic structures", Chem. Mater. 12, pp. 2838-2841, 2000.

9. G. S. He, T. C. Lin, Y. Cui, P. N. Prasad, D. Brousmiche, J. M. Serin and J. M. J. Fréchet, "Two-photon excited intramolecular energy transfer and light-harvesting effect in novel dendritic systems", Opt. Lett. 28, pp. 768-770, 2003.

10. M. Drobizhev, A. Karotki, Y. Dzenis, A. Rebane, Z. Suo and C.W. Spangler, "Strong cooperative enhancement of two-photon absorption in dendrimers" J. Phys. Chem. B 107, pp. 7540-7543, 2003.

11. D. Brousmiche, J. M. Serin, J. M. J. Fréchet, G. S. He, T. C. Lin, S. J. Chung and P. N. Prasad, "Fluorescence energy transfer in a novel two-photon absorbing system”, J. Am. Chem. Soc. 125, pp. 1448-1449, 2003.

12. L. Porrès, C. Katan, O. Mongin, T. Pons, J. Mertz and M. Blanchard-Desce, "Two-photon absorption and fluorescence in nanoscale multipolar chromophores: effect of dimensionality and charge-symmetry", J. Mol. Struct. 704, pp. 17-24, 2004.

13. M. Drobizhev, A. Rebane, Z. Suo and C.W. Spangler, "One-, two- and three-photon spectroscopy of $\pi$-conjugated dendrimers: cooperative enhancement and coherent domains", J. Lumin. 111, pp. 291-305, 2005.

14. C. Katan, F. Terenziani, O. Mongin, M. H. V. Werts, L. Porrès, T. Pons, J. Mertz, S. Tretiak and M. Blanchard Desce, "Effects of (multi)branching of dipolar chromophores on photophysical properties and two-photon absorption", J. Phys. Chem. A 109, pp. 3024-3037, 2005.

15. S. Zhiyong, M. Drobizhev, C. W. Spangler, N. Christensson and A. Rebane, "New fluorophores based on trifluorenylamine with very large intrinsic three-photon absorption cross sections", Org. Lett. 7, pp. 4807-4810, 2005.

16. M. A. Oar, J. A. Serin, W. R. Dichtel, J. M. J. Fréchet, T. Y. Ohulchanskyy and P. N. Prasad, "Photosensitization of singlet oxygen via two-photon-excited fluorescence resonance energy transfer in a water-soluble dendrimer", Chem. Mater. 17, pp. 2267-2275, 2005.

17. P. Allcock, R. D. Jenkins and D. L. Andrews, "Laser-assisted resonance-energy transfer", Phys. Rev. A 61, 023812, 2000.

18. D. L. Andrews and R. G. Crisp, “Optically-activated energy transfer: array implementation” J. Opt. A: Pure Appl. Opt. (in press).

19. D. L. Andrews and R. G. Crisp, "Theory of directed electronic energy transfer", J. Fluor. (in press).

20. R. G. Crisp and D. L. Andrews, "Optical switching in nano-arrays: transistor action through directed energy transfer", in these Proceedings.

21. P. Allcock and D. L. Andrews, "Two-photon fluorescence: resonance energy transfer", J. Chem. Phys. 108, pp. 3089-3095, 1998.

22. D. L. Andrews and D. S. Bradshaw, "Optically nonlinear energy transfer in light-harvesting dendrimers", J. Chem. Phys. 121, pp. 2445-2454, 2004.

23. M. I. Stockmann, "Kinetics of two-photon excitation of impurity centres in a condensed medium", Sov. Phys. JETP 60, pp. 49-57, 1984.

24. E. Fermi, Nuclear Physics, University of Chicago Press, Chicago, 1950. 
25. D. P. Craig and T. Thirunamachandran, Molecular Quantum Electrodynamics, Dover, Mineola, NY, 1998.

26. G. J. Daniels, R. D. Jenkins, D. S. Bradshaw and D. L. Andrews, "Resonance energy transfer: The unified theory revisited", J. Chem. Phys. 119, pp. 2264-2274, 2003.

27. D. L. Andrews and K. P. Hopkins, "Synergistic effects in two-photon absorption: the quantum electrodynamics of bimolecular mean-frequency absorption", Adv. Chem. Phys. 77, pp. 39-102, 1990.

28. R. D. Jenkins and D. L. Andrews, “Twin-donor systems for resonance energy transfer”, Chem. Phys. Lett. 301, pp. 235-240, 1999.

29. R. D. Jenkins and D. L. Andrews, "Three-centre systems for energy pooling: quantum electrodynamical theory", $J$. Phys. Chem. A 102, pp. 10834-10842, 1998.

30. D. L. Andrews and D. S. Bradshaw, "Virtual photons, dipole fields and energy transfer: A quantum electrodynamical approach", Eur. J. Phys. 25, pp. 845-858, 2004.

*david.andrews@physics.org 